\title{
New Methods to Measure HII Regions Diameters
}

\author{
O. Fuentes-Masip, H. O. Castañeda and C. Muñoz-Tuñón \\ Instituto de Astrofísica de Canarias, 38200-La Laguna (Tenerife) \\ $S P A I N$
}

\begin{abstract}
Observations of the giant irregular galaxy NGC 4449 are being used to study the correlations between the diameter or luminosity and the velocity dispersion of its giant $\mathrm{HII}$ regions (GHRs), understanding as GHRs those HII regions with supersonic velocity dispersions. In the central part of this galaxy the HII regions overlap, and also there is a strong, morphologically diffuse emission that permeates the main body of NGC 4449, both effects making difficult the detection, identification and separation of $\mathrm{HII}$ regions. We have developed new techniques to solve these problems, obtaining HII regions parameters equivalent to the ones that would be got if the HII regions did not overlap and if the diffuse emission did not exist.
\end{abstract}

\section{Introduction}

We are studying the linear correlations between the radii or luminosities of GHRs and their internal velocity dispersions, first discovered by Melnick (1977), and later confirmed and analyzed by several groups (Dyson 1979, Terlevich \& Melnick 1981, Skillman \& Balick 1984, Hippelein 1986, Arsenault \& Roy 1988, Tenorio-Tagle et al. 1993, etc). Several physical mechanisms have been proposed by these authors to maintain the supersonic velocity dispersions observed in GHRs and to produce the mentioned correlations.

The subject of this contribution is to present new methods to analyze the data, for obtaining the correct parameters to be able to correlate the data points.

One of the main motivations for this work is that these correlations could be eventually used as new extragalactic distance indicators, because observing the velocity dispersion of a GHR, we could derive its real radius or luminosity and comparing with the apparent ones we could obtain its distance.

A possible line of attack would be to study if the correlations are valid for the GHRs of a single galaxy, where all HII regions are at the same distance and are observed under the same observational conditions. Like this comparisons and statistical studies are easier and more reliable. The first work with these characteristics was carried out by Arsenault et al. (1990) with the spiral galaxy NGC 4321.

A Fabry-Perot interferometer is ideally suited for this kind of study, because it allows to perform bidimensional spectroscopy with high spatial and spectral resolution to cover extended sources, such as GHRs. 


\section{Available Data}

We have observed the giant irregular galaxy NGC 4449 in the emission lines of $H_{\alpha}$ and [OIII] $\lambda 5007 \dot{A}$, at the Cassegrain focus of the William Herschel Telescope of the Observatorio del Roque de los Muchachos with TAURUS-2, a Fabry-Perot imaging interferometer. Our observations consist of two data cubes for each emission line, with an overlap to check the consistency of our results. The data cubes cover the central area of this galaxy, which possesses a large density of HII regions, a very high proportion of GHRs and the nucleus of the galaxy. This galaxy is ideally suited to our purposes since it is nearby (5 Mpc away) and of an early type, with a high surface brightness and a large number of HII regions (252 catalogued by Sabbadin \& Bianchini 1979). NGC 4449 is an irregular galaxy, and as a consequence it has no structures such as spiral arms which could introduce environmental distinctions between different HII regions (for example those located in the arms relative to those in the interarms). All these characteristics provide a statistically significant sample of HII regions, easy to observe and to compare.

\section{Data Analysis}

In NGC 4449, the overlapping of HII regions in the central areas constitutes a problem to separate them and to measure their radii and luminosities. Also, the diffuse nebular emission permeating the main body of this galaxy again makes difficult the measurement of HII regions radii and luminosities and to detect faint regions. Of course, the determination of the velocity dispersions can also be affected by the precise setting of individual HII regions limits.

The software we have used to analyze our data is MATADOR ${ }^{1}$, which has revealed to be a powerful software to handle three-dimensional data.

To identify the HII regions, we used FOCAS (developed by F. Valdez at Kitt Peak National Observatory as a part of the IRAF package), to avoid subjective detections or observer dependent parameter determinations. We produced flux maps, made by collapsing the cubes in the spectral direction (that is to say: adding up all the $z$ planes of our data cube). These maps are equivalent to narrow band images on which FOCAS can be applied. In this way FOCAS identified an HII region as an area on the image being a relative maximum of flux above a given threshold (selected to be three times the sigma of the local background), with an area larger than that of the seeing disk ( 1 arcsec).

We extracted the spectra of the areas determined by FOCAS and we fitted them with single gaussians to get their velocity dispersions. We discarded those GHRs with spectra having low $\mathbf{S} / \mathbf{N}$, asymmetries or line splitting (since their line widths would not be well defined when fitted with a single gaussian). However, no correlation was observed between the sizes and the velocity dispersions of GHRs at this stage of the analysis.

We noticed that the luminosity profiles of isolated HII regions were gaussian. Of

\footnotetext{
${ }^{1}$ MATADOR has been developed by Vladimir Gabryusev and Casiana Muños-Tunón at the Instituto de Astrofisica de Canarias under the framework of the Grupo de Eatudios de Formación Eatelar (GEFE)
} 


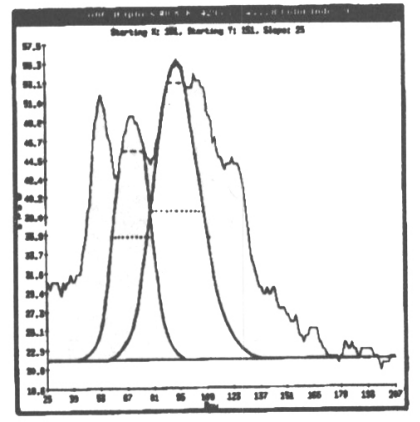

Figure 1. Example of extrapolated luminosity profile

course real HII region luminosity profiles are not gaussian, but in our case, due to the smoothing we performed to the data to increase the $S / N$, and due to the convolution with the seeing profile, luminosity profiles were really gaussian. We assumed then that all HII regions present in our data had gaussian luminosity profiles and so they could be extrapolated till a constant background determined in large areas in the perifery of our field (where there is no galactic emission), to avoid the problems derived from the overlapping and from the diffuse emission, as can be seen in Figure 1. In this figure we have plotted as a solid line a selected luminosity profile (where the above mentioned problems are evident), as a dashed line the diameter determined by FOCAS and as a dotted line the FWHM of the extrapolated gaussian, which will be the diameter we will adopt hereafter. In Figure 2 there is our flux map. The contours show the areas of the HII regions determined by FOCAS and the circles indicate the extrapolated diameters for those GHRs with symmetric, no-splitted, high $\mathrm{S} / \mathrm{N}$ spectra. This new set of diameters (once corrected for seeing broadening) are clearly correlated with the velocity dispersions (corrected for instrumental and thermal broadening), as seen in Figure 3, corresponding to our [OIII] $\lambda 5007 \dot{A}$ data.

This kind of analysis has proved valuable since in this way we have obtained significantly correlated data. In an immediate future we will compare our correlation with the previously published ones and we will study which can be the responsible physical mechanism between those proposed in the literature (Fuentes-Masip et al. 1994). We plan also to observe other nearby giant irregular galaxies to have a statistically more complete sample and to compare the results for different galaxies.

\section{Final Remarks}

1. To carry out this kind of studies we propose to use observations of a single galaxy when possible, to avoid uncertain distance determinations and differences in detection levels and resolution, due to different observational techniques, galactic environments and target distances.

2. Objective criteria must be used for the detection, separation and measurement of HII regions. The extrapolation of luminosity profiles when there 


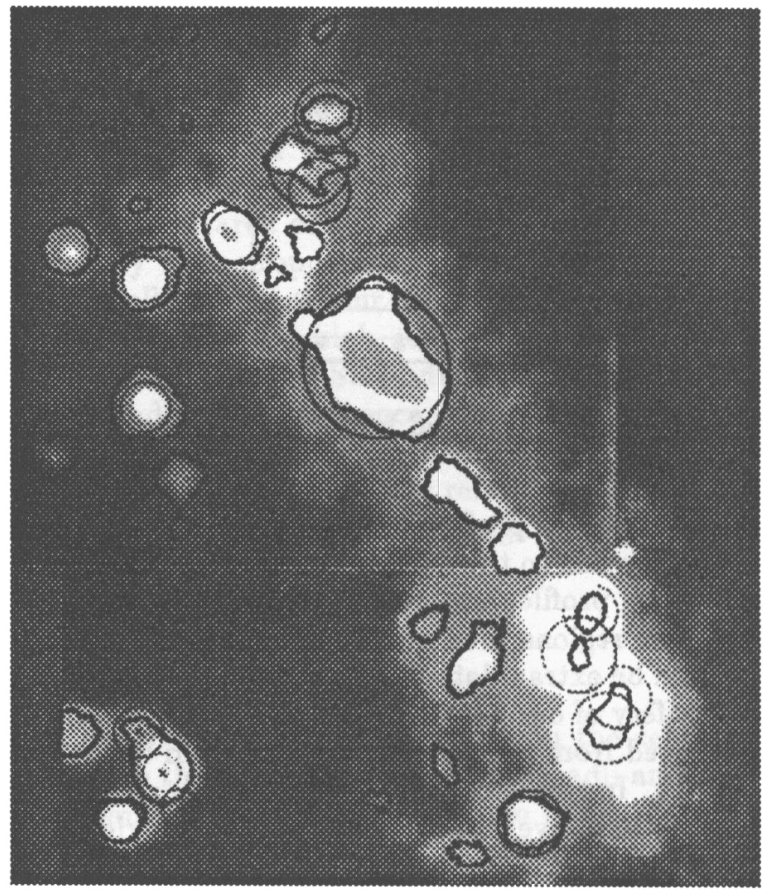

Figure 2. Flux map of NGC 4449, including the areas determined by FOCAS and the extrapolated GHRs sizes

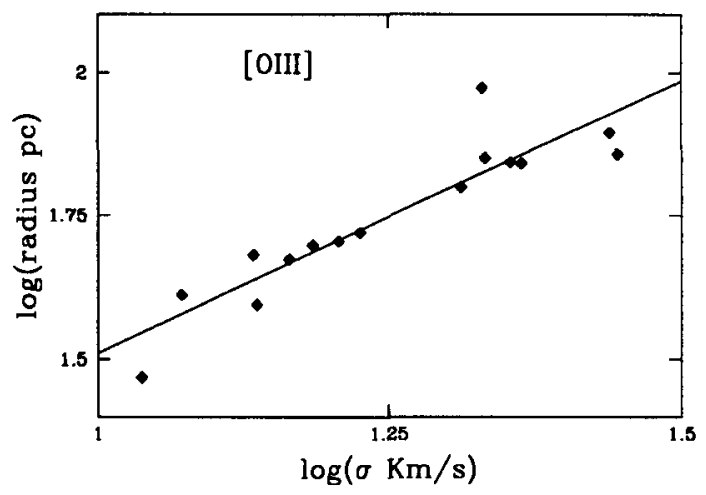

Figure 3. Resultant correlation between radii and velocity dispersions for the [OIII] $\lambda 5007 \stackrel{A}{\text { data }}$ 
is an intense nebular background or an important overlapping has also proved useful.

3. MATADOR has shown to be a powerful software to analyze three-dimensional data. It will soon be available to the scientific community.

Acknowledgments. We are grateful to E. Telles for a seminal idea about this work and to M. Serra-Ricart for his help with FOCAS.

\section{Discussion}

Dr. Hippelein: Would not it help to discriminate between neighbouring HII regions to use the single Fabry-Perot frames instead of the collapsed (flux) image?

Mr. Fuentes-Masip: The radial velocity differences between regions are not large enough to clearly separate the HII regions in different Fabry-Perot planes, but we are working in a more sophisticated method to do so, based on the radial velocity and velocity dispersion gradients between adjacent $\mathrm{HII}$ regions.

$D r$. Deharveng: Have you checked for a correlation between the velocity dispersion and the $H_{\alpha}$ luminosity? The [OIII] $\lambda 5007 \AA$ luminosity depends strongly on the stellar content of the region; thus I would expect a better correlation with the $H_{\alpha}$ luminosity.

Mr. Fuentes-Masip: We have indeed obtained a clear correlation between the velocity dispersion and the luminosity in our $H_{\alpha}$ data, but the correlation coeficient is lower ( 0.69 for $H_{\alpha}$ and 0.91 for [OIII]), probably because the sensitivity of the detector is lower at this wavelength and so our $H_{\alpha}$ data have a lower $\mathrm{S} / \mathrm{N}$.

\section{References}

Arsenault, R., \& Roy, J.R. 1988, A\&A, 201, 199

Arsenault, R., Roy, J.R., \& Boulesteix, J. 1990, A\&A, 234, 23

Dyson, J.E. 1979, A\&A, 73, 132

Fuentes-Masip, O., Muñoz-Tuñón, C., \& Castañeda, H.O. 1994 in preparation Hippelein, H. 1986, A\&A, 160, 374

Melnick, J. 1977, ApJ, 213, 15

Sabbandin, F., \& Bianchini, A. 1979, PASP, 91, 280

Skillman, E.D., \& Balick, B. 1984, ApJ, 280, 580

Tenorio-Tagle, G., Munoz-Tunon, C., \& Cox, D.P. 1993, ApJ, 418, 767

Terlevich, R., \& Melnick, J. 1981, MNRAS, 195, 839 